\title{
Loss of atypical chemokine receptor 4 facilitates C-C motif chemokine ligand 21-mediated tumor growth and invasion in nasopharyngeal carcinoma
}

\author{
YUNHE JU $^{1 *}$, CHUANZHENG SUN ${ }^{2 *}$ and XIAOLI WANG ${ }^{1}$ \\ Departments of ${ }^{1}$ Radiotherapy and ${ }^{2}$ Head and Neck Surgery, The Third Affiliated Hospital of Kunming Medical University, \\ Tumor Hospital of Yunnan Province, Kunming, Yunnan 650000, P.R. China
}

Received June 14, 2017; Accepted May 2, 2018

DOI: $10.3892 /$ etm.2018.7007

\begin{abstract}
Nasopharyngeal carcinoma (NPC) is a common malignant disease that is prevalent in Asian countries. Atypical chemokine receptor 4 (ACKR4) binds to various chemokines, including C-C motif chemokine ligand (CCL)19, CCL21, CCL25 and $\mathrm{C}-\mathrm{X}-\mathrm{C}$ motif chemokine ligand 13 , without inducing downstream signaling transduction. However, the role of ACKR4 in modulating NPC development remains unclear. In the present study, the effects of ACKR4 on NPC growth, invasion and metastasis were investigated, as well as the endogenous mechanisms through which ACKR4 mediates NPC development. The results demonstrated that ACKR4 was downregulated in human NPC tumor tissues, as compared with that in adjacent normal tissue. In a subcutaneous tumor animal model, the knockdown of ACKR4 enhanced NPC invasion and metastasis. Furthermore, CCL21 was accumulated in ACKR4 knockdown tumors. In vitro, the loss of ACKR4 increased CCL21-mediated SUNE-1 cell proliferation, epithelial-mesenchymal transition and invasion. In conclusion, the loss of ACKR4 promoted CCL21-mediated NPC development; thus, neutralizing CCL21 in NPC with low ACKR4 expression may be a novel treatment strategy.
\end{abstract}

\section{Introduction}

Nasopharyngeal carcinoma (NPC) is a common cancer type worldwide, with $>60,000$ new cases reported in China each year (1). The treatment of NPC largely relies on early detection and a combination of chemotherapy and radiotherapy $(2,3)$.

Correspondence to: Dr Xiaoli Wang, Department of Radiotherapy, The Third Affiliated Hospital of Kunming Medical University, Tumor Hospital of Yunnan Province, 519 Kunzhou Road, Kunming, Yunnan 650000, P.R. China

E-mail: yao_yl23@126.com

*Contributed equally

Key words: atypical chemokine receptor, nasopharyngeal carcinoma, growth, invasion, metastasis
However, only a small proportion of NPC cases are detected sufficiently early to be eligible for curative treatment (2). Understanding the pathogenesis of NPC is critical in order to identify novel treatment targets for this disease.

The initiation and development of NPC is a multistep process that is manipulated by cumulative genetic and epigenetic alterations, as well as microenvironmental cues (2). Atypical chemokine receptors (ACKRs) are chemokine receptors that bind to their ligands with a high affinity, but cannot induce downstream signal transduction (4,5). Although ACKRs are structurally unable to initiate signal transduction, they serve a non-redundant role in keeping the homeostasis of inflammatory responses by efficiently internalizing chemokines and maintaining chemotactic gradients $(4,5)$. As a new member of the ACKR family, ACKR4 (also known as C-C motif chemokine receptor-like 1) is usually downregulated in cancer tissues compared with normal tissues (6-8). In breast, cervical and colorectal cancer, low expression of ACKR4 was correlated with poor prognosis $(6,8,9)$. ACKR4 binds to homeostatic chemokines, including $\mathrm{C}-\mathrm{C}$ motif chemokine ligand (CCL)19, CCL21, CCL25 and C-X-C motif chemokine ligand $(\mathrm{CXCL}) 13$, which are the major ligands of $\mathrm{CC}$ receptor (CCR)7, CCR9 and CXC receptor (CXCR)5, thereby tuning up the function of these chemokine axes $(4,5)$.

Inflammatory responses have been reported to regulate the development of NPC (10). It is well established that the CCL21/CCR7 axis promoted cancer development via enhancing the proliferation, migration and invasion of tumor cells $(11,12)$. ACKR4-mediated scavenging of dermal-derived CCL21/CCR7 is critical during inflammation, Thus, the effect of ACKR4 on NPC cells during inflammation, facilitates CCR7-dependent cell trafficking by scavenging CCL21 (12). However, the effects of ACKR4 in regulating inflammation-mediated NPC development, particularly the CCL21/CCR7 chemokine-axis related, remain unclear. In the present study, it is hypothesized that loss of ACKR4 in NPC is an important promoting factor of the CCL21-mediated tumor growth and invasion.

\section{Materials and methods}

Cell culture and transfection. The mouse NPC cell line SUNE-1 was purchased from the American Type Culture 
Collection (Manassas, VA, USA). Cells were cultured in Dulbecco's modified Eagle's medium with high glucose, supplemented with $10 \%$ fetal bovine serum, $100 \mathrm{IU} / \mathrm{ml}$ penicillin $\mathrm{G}$ and $100 \mathrm{mg} / \mathrm{ml}$ streptomycin (all from Thermo Fisher Scientific, Inc., Waltham, MA, USA), at $37^{\circ} \mathrm{C}$ in a humidified incubator containing $5 \% \mathrm{CO}_{2}$.

For the overexpression of ACKR4 in the SUNE-1 cell line, Human ACKR4 cDNA plasmids (obtained from OriGene Technologies, Inc., Rockville, MD, USA) were transfected into 293 cells $\left(1 \times 10^{6} /\right.$ well) for $48 \mathrm{~h}$ to generate a lentivirus using Lipofectamine ${ }^{\circledR} 2000$ (Invitrogen; Thermo Fisher Scientific, Inc.) according to the manufacturer's protocol. The viral supernatant was subsequently collected and used to infect the SUNE-1 cells. Further analysis was performed $72 \mathrm{~h}$ post-transfection.

SUNE-1 Cells were transfected with specific siRNA (5'-GUUCUGCAGCAACAUUUAAUU-3') against ACKR4 using Lipofectamine 2000 (Invitrogen; Thermo Fischer Scientific, Inc.) according to the manufacturer's Protocol. Cells were seeded into 6-well plates and cultured for an additional $24 \mathrm{~h}$ at $37^{\circ} \mathrm{C}$. The medium was refreshed with serum-free RPMI 1640 medium (Thermo Fisher Scientific, Inc.) and siRNA using Lipofectamine 2000 (Invitrogen, Carlsbad, CA) according to the protocol, and were added into each well. Following $6 \mathrm{~h}$, the medium was replaced with normal RPMI 1640 medium supplemented with $10 \%$ fetal bovine serum (Gibco; Thermo Fisher Scientific, Inc.). Cells were harvested for subsequent analysis $48 \mathrm{~h}$ following transfection in order to collect total RNAs or proteins. siRNAs were synthesized by Boute Biotech Co. Ltd., (Wuhan, China). The empty backbone vector was used as a transfection control. Flow cytometry was then conducted to validate the upregulation or downregulation of membrane ACKR4 expression.

Patients and tissues. A total of 30 patients (age, 30-75 years; male:female, 14:16) who were diagnosed with nasopharyngeal carcinoma and received radical surgery for this at the Department of Head and Neck of The Third Affiliated Hospital of Kunming Medical University (Kunming, China) were included in the present study. All patients were admitted between January 2015 and January 2016 and were not treated with chemotherapy or radiotherapy prior to surgery. Patients were included in the present study if they: i) Were pathologically diagnosed with NPC; ii) were aged $18-80$ years old; iii) signed the written informed consent form provided by the hospital medical ethics committee of the Third Affiliated Hospital of Kunming Medical University. Cancerous tissues and matched adjacent non-cancerous tissues $(3 \mathrm{~cm}$ away from the tumor) were harvested from each patient following surgery. The present study was approved by the Research Ethics Committee of Third Affiliated Hospital of Kunming Medical University. Tumor tissues were fixed using 10\% formalin solution for $12 \mathrm{~h}$ at $4^{\circ} \mathrm{C}$, paraffin embedded and sliced to $4 \mu \mathrm{m}$ thick sections. Epitope retrieval was then performed for further analysis.

Flow cytometry analysis. Flow cytometry was used to measure ACKR4 expression in human fresh NPC patient samples and SUNE-1 cells. Briefly, tumor and adjacent normal tissues were collected, and digested with $1 \mu \mathrm{g} / \mathrm{ml}$ type IV collagenase and 20 units/ml DNase type IV (both from
Thermo Fisher Scientific, Inc.) in Hank's balanced salt solution. Subsequently, the isolated single cells from the tissues were stained with fluorochrome-conjugated anti-ACKR4 antibody (1:500; cat. no. TA340600; Origene technologies, Inc.) without permeabilization and then incubated for $2 \mathrm{~h}$ at room temperature with mouse anti goat $\mathrm{H} \& \mathrm{~L}$ secondary antibodies (1:1,000; cat. no. ab7064; Abcam, Cambridge, UK) Samples were analyzed by a BD LSRFortessa ${ }^{\mathrm{TM}}$ cell analyzer (BD Biosciences, Franklin Lakes, NJ, USA). The stained cells were analyzed using a Becton Dickinson FACScan flow cytometer using WinMD Isoftware (version 2.9; BD Biosciences).

Invasion assay. Transwell chambers coated with Matrigel (Corning, Inc., Corning, NY, USA) with an $8-\mu$ m polycarbonate filter membrane were used to perform the invasion assay. Briefly, SUNE-1 cells cultured in 6 well pates $\left(3 \times 10^{6} /\right.$ well) with different ACKR4 levels were added into the upper chamber along with serum-free medium. The lower chamber was filled with 5\% serum medium, and the plate was incubated under normal conditions for $24 \mathrm{~h}$. Subsequently, the cells remaining on the top surface of the filter were removed, and the cells that had invaded into the lower part of the filter were fixed for $20 \mathrm{~min}$ in paraformaldehyde at room temperature and stained with $0.1 \%$ crystal violet for $20 \mathrm{~min}$ at room temperature. The stained membrane was then washed with PBS for three times. To quantify the amount of invading cells, cell count was performed on each membrane.

Cell counting assay. Cell Counting Kit-8 (CCK-8; Dojindo Molecular Technologies, inc., Kumamoto, Japan) was used to estimate the cell number according to the manufacturer's protocol. Briefly, the same number of SUNE-1 cells $\left(5 \times 10^{5} /\right.$ well $)$ different levels of ACKR4 expression was seeded in 96-well plates. The number of cells in each well was counted at $0,6,12,24$ and $48 \mathrm{~h}$ after seeding. At these time points, CCK- 8 solution was added and incubated for $30 \mathrm{~min}$ at $37^{\circ} \mathrm{C}$, and then the absorbance at $450 \mathrm{~nm}$ was measured by an MRX II microplate reader (Dynex Technologies, Chantilly, VA, USA).

Animal model. A total of 32 subcutaneous NPC model was established using 5-week-old female NUDE mice (weight, 18-20 g; Shanghai SLAC Laboratory Animal Co., Ltd., Shanghai, China) with SUNE-1 cells expressing different levels of ACKR4 achieved by transfection with wild-type, empty control, knockdown and overexpression vectors, respectively. All mice were bred under a specific pathogen-free environment with a controlled environment (temperature, $23 \pm 1^{\circ} \mathrm{C}$; humidity, $50-60 \%$ ) with an artificial simulation of 12 -h light-dark cycle, standard food, and free access to autoclaved water. Mice were acclimatized prior to experimentation for 3 days. A total of $5 \times 10^{5}$ SUNE-1 cells were inoculated subcutaneously to the flanks of the mice, and the tumor size and body weight were measured daily. The tumor volume was calculated according to the following formula: Tumor volume $=$ length $\mathrm{x}$ width ${ }^{2} \mathrm{x} 0.5$. In total, 8 mice were included in each group.

Reverse transcription-quantitative polymerase chain reaction $(R T-q P C R)$. To measure the expression levels of various epithelial-mesenchymal transition (EMT)-associated genes 

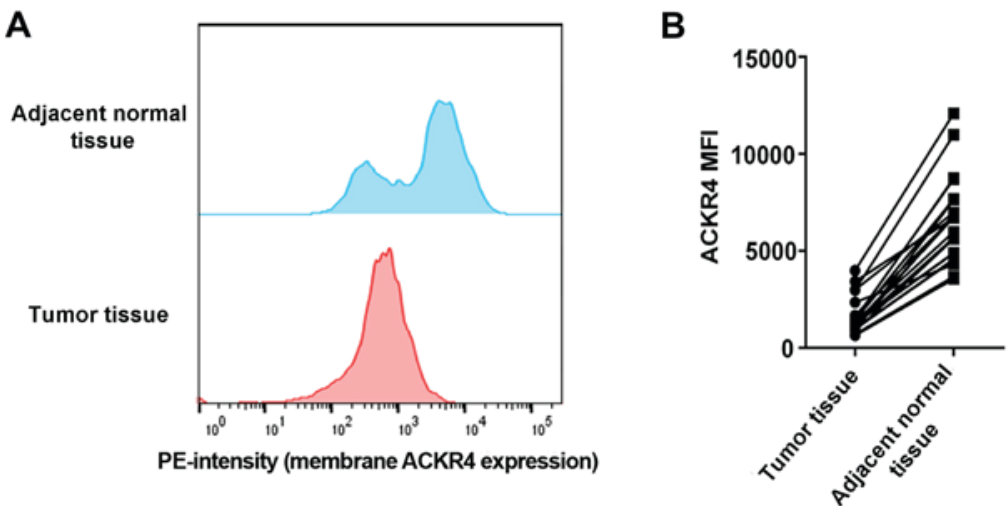

Figure 1. Expression of ACKR4 in NPC and adjacent normal tissues. (A) Representative plots showing the ACKR4 expression level in human NPC and adjacent tissues. (B) Comparison of ACKR4 expression in paired NPC and adjacent tissues. A total of 15 NPC and paired adjacent samples were tested. ACKR4, atypical chemokine receptor 4; NPC, nasopharyngeal carcinoma; MFI, mean fluorescence intensity; PE, Phycoerythrin.

in SUNE-1 cells, the EMT PCR Array for RT-qPCR analysis (Qiagen, Hilden, Germany) was used. Initially, the mirVana miRNA Isolation kit (Thermo Fisher Scientific, Inc.) was used to extract total RNA from the cells, which was then reverse transcribed to cDNA using the miScript Reverse Transcription kit (Qiagen). Next, qPCR was performed according to the protocol described in the EMT PCR Array kit. The primers were designed as follows: Snail family transcriptional repressor 1 (SNAI-1) forward, 5'-CAAAGGTGGATCAGA TTCAAG-3' and reverse, 5'-GGTGAGCATTATCACCCA GAA-3'; Fibronectin 1 (FN1) forward, 5'-CAAAGGTGGATC AGATTCAAG-3' and reverse, 5'-GGTGAGCATTATCAC CCAGAA-3'; Variant in Methylation (VIM) forward, 5'-AGA AACCGGCAGAGTGCTCTTA-3' and reverse, 5'-GTACCC AGGCAACAGAATCCA-3'; CAV2 forward, 5'-TGGCAC CCAGCACAATGAA-3' and reverse, 5'-CTAAGTCATAGT CCGCCTAGAAGCA-3; $\beta$-actin forward, 5'-AGAAAATCT GGCACCACACC-3' and reverse, 5'-TAGCACAGCCTGGAT AGCAA-3'. The thermocycling conditions were as follows: $95^{\circ} \mathrm{C}$ for $5 \mathrm{~min}$, followed by 35 cycles at $95^{\circ} \mathrm{C}$ for $20 \mathrm{sec}, 58^{\circ} \mathrm{C}$ for $20 \mathrm{sec}$ and $72^{\circ} \mathrm{C}$ for $20 \mathrm{sec}$, with a final extension at $72^{\circ} \mathrm{C}$ for $5 \mathrm{~min} . \beta$-actin was used as the internal reference gene. The top four differentially expressed genes were selected and plotted in a barplot. Relative gene expression was determined by the comparative quantitative cycle (Cq) method (13).

Furthermore, to detect lymphatic metastasis in the animal model following inoculation for 50 days, the human 18srRNA level in the tumor-draining lymph nodes of the mice was measured by RT-qPCR assay as described previously, with the following primers: Forward, 5'-GGCCCTGTA ATTGGAATGAGTC-3', and reverse, 5'-CCAAGATCCAACTACGAGCTT-3'. The tumor-draining lymph nodes with positive human 18srRNA expression were considered to be tumor metastasis-positive.

ELISA. An enzyme-linked immunosorbent assay (ELISA) was used to measure the concentrations of CCL19 (cat. no. ab100601; Abcam, Cambridge, UK), CCL21 (cat. no. ab208985; Abcam), CCL25 (cat. no. ab100645; Abcam), CXCL13 (cat. no. ab179881; Abcam), matrix metalloproteinase (MMP)2 (cat. no. ab100606; Abcam) and MMP9 (cat. no. ab100610; Abcam) in the cell lysate or tumor tissues.
The samples from mice were collected after inoculation for 50 days and samples from cells were collected after inoculation for $48 \mathrm{~h}$. All ELISA kits were purchased from Thermo Fisher Scientific, Inc. (eBioscience), and the experiments followed the manufacturer's protocol. The total protein concentration was normalized to the concentration in the samples prior to incubation with vectors or to inoculated animals.

Statistical analysis. GraphPad Prism software (Version 7.0; GraphPad Software, Inc., La Jolla, CA, USA) was used for statistical analysis and data visualization. Comparisons were analyzed by t-test if the results were presented as quantitative data. $\chi^{2}$ analysis was used for frequency data. The Kaplan-Meier method and log-rank test were used for survival analysis and for evaluating the difference between different cohorts, respectively. A two-tailed P-value of $<0.05$ was considered to be an indicator of a statistically significant difference.

\section{Results}

ACKR4 downregulation in NPC tissues. To understand the role of ACKR4 in NPC development, the current study first investigated whether ACKR4 was dysregulated in human NPC cancer tissue compared with its level in adjacent normal liver tissue. Via flow cytometry analysis, it was observed that the expression of ACKR4 was lower in NPC tissues as compared with that in adjacent normal tissues (Fig. 1A and B). These data suggested that ACKR4 was abnormally downregulated in NPC cells, providing the rationale to manipulate ACKR4 expression in NPC cell lines and animal models.

Loss of ACKR 4 promotes tumor development and lymph node metastasis in an NPC animal model. ACKR4 expression was upregulated or downregulated in SUNE-1 cells (Fig. 2A and B). Subsequently, a subcutaneous tumor animal model, in which different levels of ACKR4 were expressed, was established by inoculation with these cells (Fig. 2C). As shown in Fig. 2C, downregulation of ACKR4 significantly accelerated the tumor growth rate, while tumors with high ACKR4 expression exhibited delayed tumor growth (Fig. 2C). Lymph node metastasis of these tumors was further investigated, and 
A

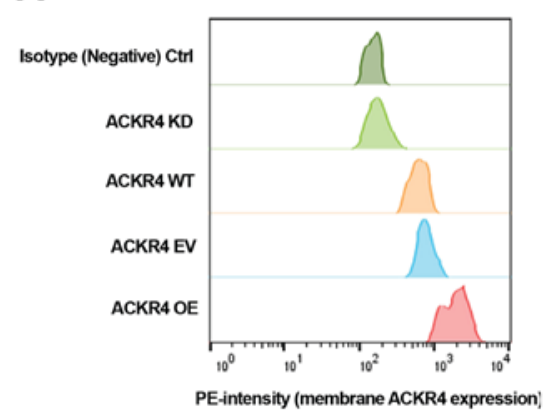

B

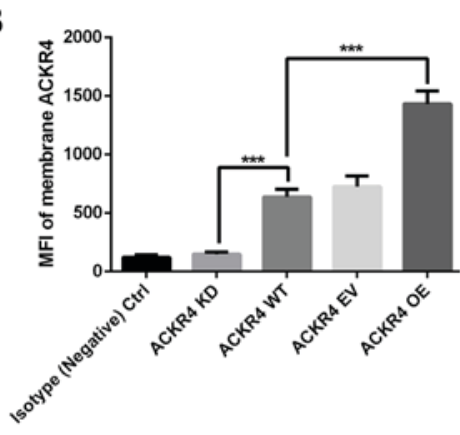

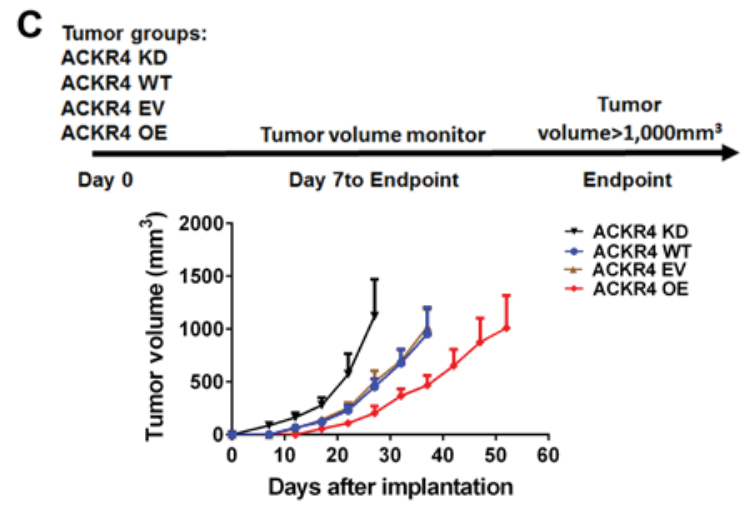

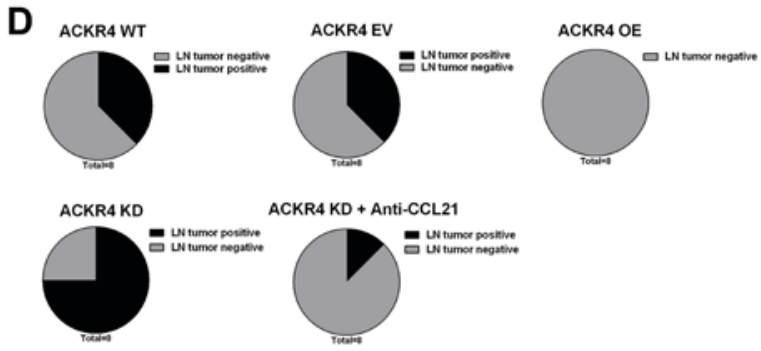

LN metastasis at end point

Figure 2. ACKR4 expression and nasopharyngeal carcinoma development in a subcutaneous SUNE-1 tumor animal model Via flow cytometry analysis, (A) quantitative analysis of expression of ACKR4 (B) ACKR4 expression level was modulated by its expression vector and small hairpin RNA in SUNE-1 cells to achieve overexpression and knockdown, respectively. The membrane ACKR4 expression was measured and plotted. (C) Experimental plan of the animal model and tumor growth curves. (D) At the endpoint, tumor metastasis in tumor-draining lymph nodes was measured, with the ACKR4 knockdown group exhibiting the highest tumor lymph node metastasis frequency. ${ }^{* * *} \mathrm{P}<0.001$. ACKR4, atypical chemokine receptor 4; MFI, mean fluorescence intensity; WT, wild-type; EV, empty vector; KD, knockdown; OE, overexpression; CCL21, C-C motif chemokine ligand 21.

loss of ACKR4 was observed to promote tumor cell lymph node metastasis (Fig. 2D). Taken together, the present in vivo study indicated that the loss of ACKR4 promoted NPC development and lymph node metastasis.

Loss of ACKR4 leads to CCL21 accumulation in NPC tissues. ACKR4 exerts its biological function by scavenging CCL19, CCL21, CCL25 and CXCL13 (12). Among these chemokines, ACKR4 exhibits the highest affinity for CCL21 (12). The concentrations of CCL19, CCL21, CCL25 and CXCL13 in the patient tumor tissue lysates were measured. As shown in Fig. 3A, loss of ACKR4 in NPC tumors led to significant accumulation of CCL21 in the tumor tissue. By contrast, overexpression of ACKR4 markedly reduced CCL21 concentration in NPC tumor tissue
(Fig. 3A). However, the level of ACKR4 expression did not regulate the concentrations of CCL19, CCL25 and CXCL13 in the NPC tumor model (Fig. 3B-D). These data suggested that CCL21 mediated the tumor development caused by ACKR4 downregulation.

Loss of ACKR4 enhances SUNE-1 cell proliferation in vitro. Given that the loss of ACKR4 promoted SUNE-1 tumor development in vivo, the present study further hypothesized that loss of ACKR4 may directly enhance SUNE-1 cell proliferation. In vitro assays revealed that SUNE-1 cells with downregulated ACKR4 expression grew much faster in comparison with SUNE-1 cells with wild-type ACKR4 expression (Fig. 4A). An ELISA assay was also performed to validate that the cell proliferation rate was changed 
A

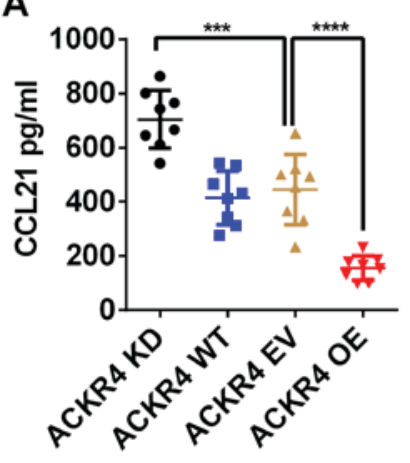

C

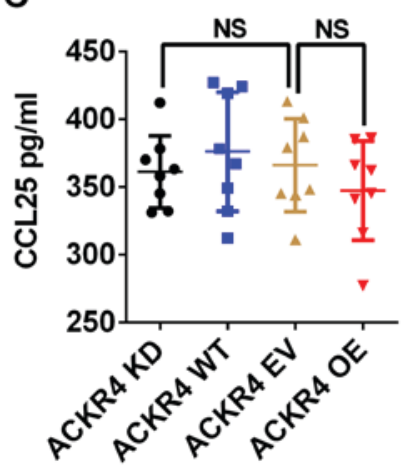

B

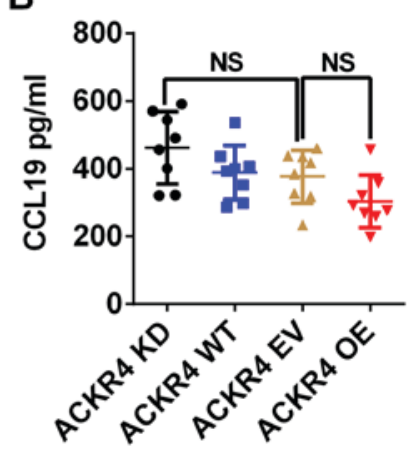

D

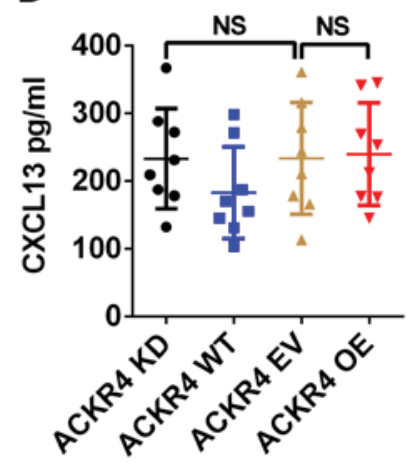

Figure 3. ACKR4 regulates CCL21 level in NPC tumor tissue. The intratumoral levels of (A) CCL21, (B) CCL19, (C) CCL25 and (D) CXCL13 were measured in tumors with different levels of ACKR4 expression. The concentration of CCL21 was evidently reduced by ACKR4 overexpression in NPC tumor tissue. ${ }^{* * *} \mathrm{P}<0.001$ and ${ }^{* * * *} \mathrm{P}<0.0001$. NS, no significant difference. ACKR4, atypical chemokine receptor 4; NPC, nasopharyngeal carcinoma; WT, wild-type; EV, empty vector; KD, knockdown; OE, overexpression CCL, C-C motif chemokine ligand; CXCL, C-X-C motif chemokine ligand.

due to ACKR4 expression (Fig. 4B). SUNE-1 cells with downregulated ACKR4 expression had the highest BrdU incorporation (Fig. 4B). Notably, when CCL21 was blocked by its neutralizing antibody, the proliferation rate of SUNE-1 cells with downregulated ACKR4 was significantly reduced (Fig. 4A and B). Taken together, these data supported that loss of ACKR4 accelerated SUNE-1 cell proliferation via a CCL21-depedent mechanism.

Loss of ACKR4 induces EMT in SUNE-1 cells. EMT is an initial event of tumor cell invasion and metastasis. As the results presented earlier indicated that loss of ACKR4 promoted tumor cell lymph node metastasis (Fig. 1G), the study further investigated whether the loss of ACKR4 induced EMT. The EMT gene signature was measured, and the top four genes with altered expression were plotted (Fig. 5). The EMT-associated genes, including VIM, FN1 and SNAI1, were upregulated in ACKR4 knockdown SUNE-1 cells, as compared with their levels in ACKR4 wild-type SUNE-1 cells (Fig. 5A-C). However, CAV2, a gene associated with epithelial function, was upregulated in ACKR4 overexpression cells (Fig. 5D). Furthermore, in the ACKR4 overexpression cells, the expression levels of three EMT-associated genes were suppressed (Fig. 5A-C). Notably, when CCL21 was blocked, the EMT gene expression was reduced in the ACKR4 knockdown
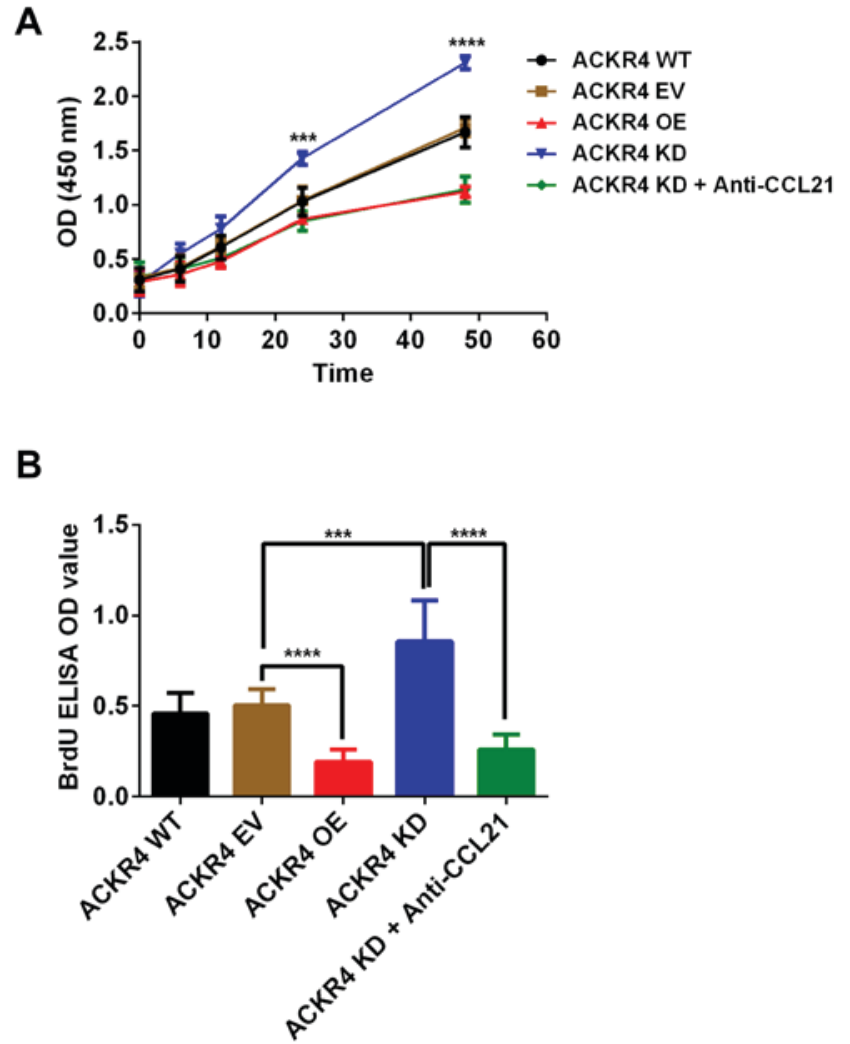

Figure 4. Effects of ACKR4 and CCL21 on SUNE-1 cell proliferation. (A) proliferation rate was counted by the cell counting kit- 8 assay. ACKR4 $\mathrm{KD}$ cells exhibited the highest rate of cell number increase. (B) Incorporation of BrdU was tested in SUNE-1 cells with different levels of ACKR4 expression. ACKR4 KD cells exhibited the highest level of BrdU incorporation, indicating their high proliferation rate. ${ }^{* * *} \mathrm{P}<0.001$ and ${ }^{* * * *} \mathrm{P}<0.0001$ vs. ACKR4 EV group. ACKR4, atypical chemokine receptor 4; WT, wild-type; EV, empty vector; KD, knockdown; OE, overexpression; CCL21, C-C motif chemokine ligand 21; OD, optical density.

SUNE-1 cells, suggesting that loss of ACKR4 induced EMT via CCL21-depedent mechanisms.

Loss of ACKR4 enhances SUNE-1 cell invasion in vitro. Since ACKR4 knockdown upregulated the EMT genes in SUNE-1 cells, the role of ACKR4 on SUNE-1 cell invasion was further examined. As shown in Fig. 6, loss ACKR4 expression improved the invasion ability of SUNE-1 cells, while overexpression of ACKR4 reduced invasion. MMP2 and MMP9 are the two key MMPs that mediate tumor cell invasion. As expected, loss of ACKR4 induced MMP2 and MMP9 expression in SUNE-1 cells (Fig. 6C and D), supporting that the loss of ACKR4 was able to improve SUNE-1 cell invasion ability. Furthermore, by blocking CCL21, tumor invasion caused by ACKR4 knockdown was suppressed in SUNE-1 cells.

\section{Discussion}

Chemokines are involved in almost all pathologies, including cancer (14). In addition, the inflammatory response has been recognized as a constitutive hallmark of cancer (14). Over the past two decades, ACKRs have been identified as a significant component of the inflammatory response $(4,5)$, efficiently 
A

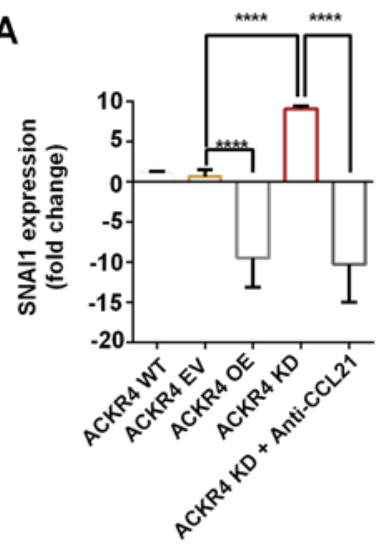

C

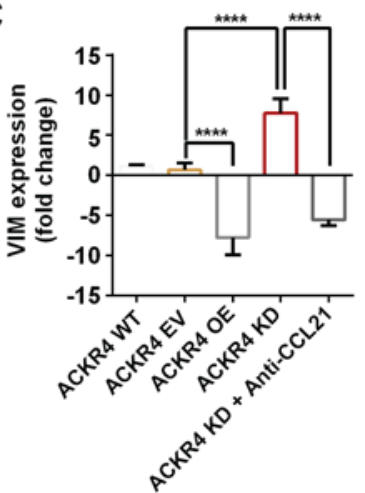

B
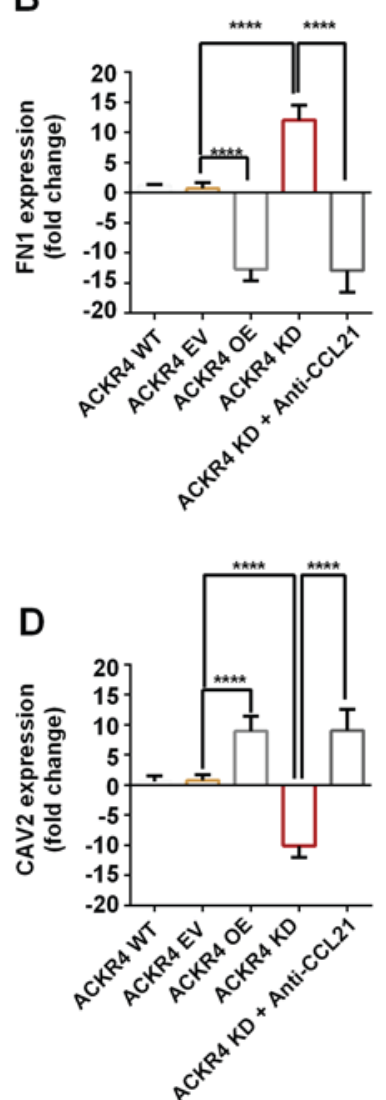

Figure 5. Expression of EMT-associated genes in nasopharyngeal carcinoma cells with different ACKR4 expression levels. The four plotted genes exhibited the highest fold change between the ACKR4 OE and ACKR4 KD cells out of all the EMT-associated genes examined. (A) SNAIL1, (B) FN1 and (C) VIM were upregulated in ACKR4 KD cells, but were downregulated in ACKR4 OE cells. (D) CAV2 gene exhibited the opposite trend. ${ }^{* * * * *} \mathrm{P}<0.0001$. EMT, epithelial-mesenchymal transition; ACKR4, atypical chemokine receptor 4; WT, wild-type; EV, empty vector; KD, knockdown; OE, overexpression; CCL21, C-C motif chemokine ligand 21. VIM, Variant in Methylation; FN1, Fibronectin 1; SNAI1, snail family transcriptional repressor 1.

\section{A}
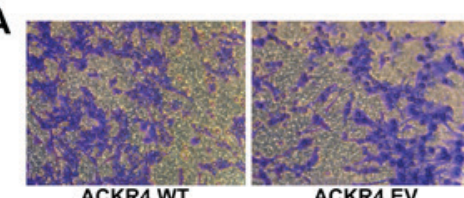

ACKR4 EV
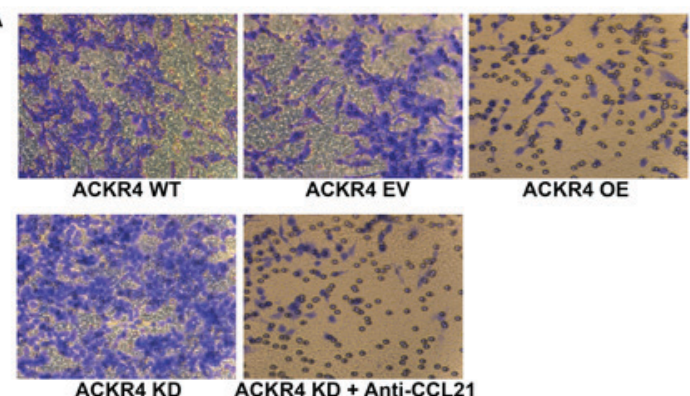

ACKR4 KD

C

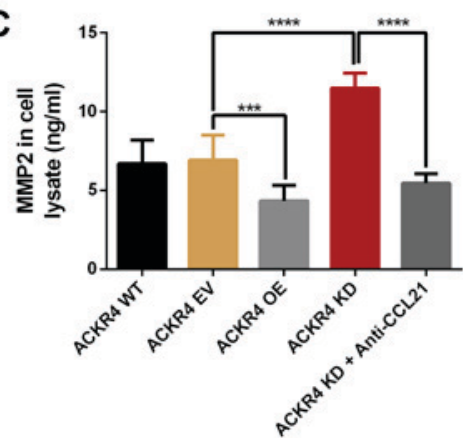

ACKR4 OE

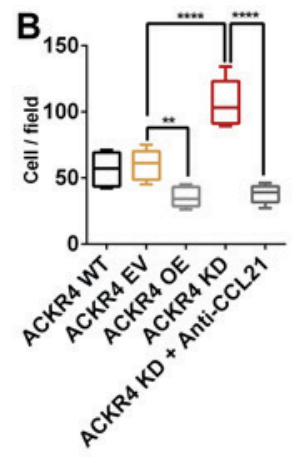

D

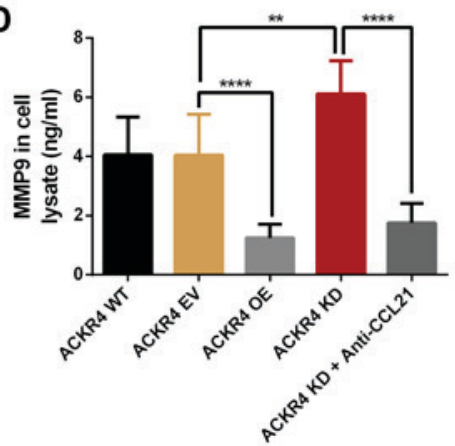

Figure 6. ACKR4 expression and nasopharyngeal carcinoma SUNE-1 cell invasion. (A) Representative figures of cell invasion assay (magnification, x200), (B) ACKR4 OE cells and anti-CCL21-treated ACKR4 KD cells displayed decreased cell invasion, whereas ACKR4 KD cells exhibited elevated cell invasion. Expression levels of (C) MMP2 and (D) MMP9 were increased in ACKR4 KD cells. ${ }^{* *} \mathrm{P}<0.01,{ }^{* * * *} \mathrm{P}<0.001$ and ${ }^{* * * * *} \mathrm{P}<0.0001$. ACKR4, atypical chemokine receptor 4; WT, wild-type; EV, empty vector; KD, knockdown; OE, overexpression; CCL21, C-C motif chemokine ligand 21; MMP, matrix metalloproteinase. 
decreasing the chemokine concentration in inflamed tissues via internalization $(4,5)$. As a member of the ACKR family, ACKR4 internalizes CCL19 and CCL21 with a high efficiency once bound to them $(4,5)$. CCL19 and CCL21 are also the two sole ligands to CCR7, which has a critical impact on regulating tumor biology (15). However, there is limited information on the pathological functions of ACKR4 in NPC.

To examine the effects of ACKR4 on NPC development, the present study first measured ACKR4 expression in NPC clinical samples. Notably, ACKR4 was significantly downregulated in the tumor tissue, when compared with the adjacent normal tissue. Meanwhile, knockdown of ACKR4 in NPC cells significantly increased tumor growth and lymph node metastasis. These observations were in line with those of previous studies in breast cancer, colorectal cancer and NPC, which reported that ACKR4 was abnormally downregulated in tumors $(6,9,16)$. Therefore, these findings suggested that loss of ACKR4 is a promoting factor of NPC.

ACKR4 has been reported to be a scavenger of CCL19, CCL21, CCL25 and CXLC13 (4,5). To determine the mechanism by which loss of ACKR4 promotes NPC development, the scavenging ability of ACKR4 to its ligands was measured in the NPC cell condition. ACKR4 was found to efficiently decrease CCL21 concentration in NPC tumor tissue. It is known that overexpression of CCL21 promoted tumor proliferation, invasion and immune suppression in tumor models $(17,18)$. In the present in vitro experiments, when CCL21 was neutralized, knockdown of ACKR4 did not exhibit any tumor promoting effects. Thus, in NPC, accumulation of CCL21 in tumor tissue may be the major mechanisms through which loss of ACKR4 promotes tumor development.

It has been reported that the CCL21/CCR7 signaling pathway enhances transforming growth factor- $\beta 1$-dependent EMT, which is a major mechanism of tumor invasion and metastasis (19). In light of this fact, the current study further explored the role of ACKR4 in reducing EMT in NPC. Loss of ACKR4 significantly upregulated EMT gene expression and tumor cell invasion in vitro. When ACKR4 was overexpressed or CCL21 was neutralized, EMT genes were significantly downregulated, and the invasion ability of tumor cells was reduced in vitro. Altogether, these key observations supported that overexpression of ACKR4 impaired CCL21/CCR7 mediated EMT and tumor cell invasion.

In conclusion, the present study investigated the effects of ACKR4 on tumor development in the NPC system. The findings of this study have provided evidence that loss of ACKR4 in NPC is prevalent and may promote NPC development via accumulating CCL21 in tumor tissue. Therefore, neutralizing CCL21 in NPC with low ACKR4 expression may be a novel treatment that requires further investigation.

\section{Acknowledgements}

Not applicable.

\section{Funding}

The present study was supported by an internal grant from the Third Affiliated Hospital of Kunming Medical University (Kunming, China).

\section{Availability of data and materials}

The analyzed data sets generated during the present study are available from the corresponding author on reasonable request.

\section{Authors' contributions}

XW and CS designed the present study and analyzed the data; $\mathrm{CS}$ and YJ performed the experiments.

\section{Ethics approval and consent to participate}

All animal procedures and the use of human tissues were reviewed and approved by the Ethical Committee of Third Affiliated Hospital of Kunming Medical University (kunming, China) and all efforts were made to minimize the suffering of the experimental mice.

\section{Patient consent for publication}

Informed consent was obtained from all patients prior to enrollment

\section{Competing interests}

The authors declare that they have no competing interests.

\section{References}

1. Chen W, Zheng R, Baade PD, Zhang S, Zeng H, Bray F, Jemal A, $\mathrm{Yu}$ XQ and He J: Cancer statistics in China, 2015. CA Cancer J Clin 66: 115-132, 2016.

2. Chua ML, Wee JT, Hui EP and Chan AT: Nasopharyngeal carcinoma. Lancet 387: 1012-1024, 2016.

3. Blanchard P, Lee A, Marguet S, Leclercq J, Ng WT, Ma J, Chan AT, Huang PY, Benhamou E, Zhu G, et al: Chemotherapy and radiotherapy in nasopharyngeal carcinoma: An update of the MAC-NPC meta-analysis. Lancet Oncol 16: 645-655, 2015.

4. Ulvmar MH, Hub E and Rot A: Atypical chemokine receptors. Exp Cell Res 317: 556-568, 2011.

5. Massara M, Bonavita O, Mantovani A, Locati M and Bonecchi R: Atypical chemokine receptors in cancer: Friends or foes? J Leukoc Biol 99: 927-933, 2016.

6. Feng LY, Ou ZL, Wu FY, Shen ZZ and Shao ZM: Involvement of a novel chemokine decoy receptor CCX-CKR in breast cancer growth, metastasis and patient survival. Clin Cancer Res 15: 2962-2970, 2009.

7. Harata-Lee Y, Turvey ME, Brazzatti JA, Gregor CE, Brown MP, Smyth MJ, Comerford I and McColl SR: The atypical chemokine receptor CCX-CKR regulates metastasis of mammary carcinoma via an effect on EMT. Immunol Cell Biol 92: 815-824, 2014.

8. Hou T, Liang D, Xu L, Huang X, Huang Y and Zhang Y: Atypical chemokine receptors predict lymph node metastasis and prognosis in patients with cervical squamous cell cancer. Gynecol Oncol 130: 181-187, 2013.

9. Zhu Y, Tang W, Liu Y, Wang G, Liang Z and Cui L: CCX-CKR expression in colorectal cancer and patient survival. Int J Biol Markers 29: e40-e48, 2014.

10. Hernandez-Gea V, Toffanin S, Friedman SL and Llovet JM: Role of the microenvironment in the pathogenesis and treatment of hepatocellular carcinoma. Gastroenterology 144: 512-527, 2013.

11. Pang MF, Georgoudaki AM, Lambut L, Johansson J, Tabor V, Hagikura K, Jin Y, Jansson M, Alexander JS, Nelson CM, et al: TGF- $\beta 1$-induced EMT promotes targeted migration of breast cancer cells through the lymphatic system by the activation of CCR7/CCL21-mediated chemotaxis. Oncogene 35: 748-760, 2016.

12. Mo M, Zhou M, Wang L, Qi L, Zhou K, Liu LF, Chen Z and $\mathrm{Zu}$ XB: CCL21/CCR7 enhances the proliferation, migration, and invasion of human bladder cancer T24 cells. PLoS One 10: e0119506, 2015. 
13. Livak KJ and Schmittgen TD: Analysis of relative gene expression data using real-time quantitative PCR and the 2(-Delta Delta C(T)) method. Methods 25: 402-408, 2001

14. Elinav E, Nowarski R, Thaiss CA, Hu B, Jin C and Flavell RA: Inflammation-induced cancer: Crosstalk between tumours, immune cells and microorganisms. Nat Rev Cancer 13: 759-771, 2013.

15. Forster R, Schubel A, Breitfeld D, Kremmer E, Renner-Muller I, Wolf E and Lipp M: CCR7 coordinates the primary immune response by establishing functional microenvironments in secondary lymphoid organs. Cell 99: 23-33, 1999.

16. Shi JY, Yang LX, Wang ZC, Wang LY, Zhou J, Wang XY, Shi GM, Ding ZB, Ke AW, Dai Z, et al: CC chemokine receptor-like 1 functions as a tumour suppressor by impairing CCR7-related chemotaxis in hepatocellular carcinoma. J Pathol 235: 546-558, 2015.

17. Xiong Y, Huang F, Li X, Chen Z, Feng D, Jiang H, Chen W and Zhang X: CCL21/CCR7 interaction promotes cellular migration and invasion via modulation of the MEK/ERK1/2 signaling pathway and correlates with lymphatic metastatic spread and poor prognosis in urinary bladder cancer. Int J Oncol 51: 75-90, 2017.
18. Irshad S, Flores-Borja F, Lawler K, Monypenny J, Evans R, Male V, Gordon P, Cheung A, Gazinska P, Noor F, et al: ROR $\gamma \mathrm{t}+$ Innate lymphoid cells promote lymph node metastasis of breast cancers. Cancer Res 77: 1083-1096, 2017.

19. Ma H, Gao L, Li S, Qin J, Chen L, Liu X, Xu P, Wang F, Xiao H, Zhou S, et al: CCR7 enhances TGF- 31 -induced epithelial-mesenchymal transition and is associated with lymph node metastasis and poor overall survival in gastric cancer. Oncotarget 6: 24348-24360, 2015.

(c) (i) () $९$ This work is licensed under a Creative Commons Attribution-NonCommercial-NoDerivatives 4.0 International (CC BY-NC-ND 4.0) License. 